\title{
T-MYCOPLASMAS: A STUDY OF THE MORPHOLOGY, ULTRASTRUCTURE AND MODE OF DIVISION OF SOME HUMAN STRAINS
}

\author{
J. WhITESCARVER* AND G. FURNESS \\ Departments of Microbiology, College of Medicine and Dentistry of New Jersey, \\ New Jersey Medical School, and the \\ Graduate School of Biomedical Sciences, 100 Berger Street, Newark, New Jersey 07103, USA
}

\section{Plates XVIII ANd XIX}

ReLATIVELY little is known about the gross morphology, mode of division and ultrastructure of the T-mycoplasmas. In stained preparations viewed by light microscopy they appear as uniform, minute coccoid particles, about $0.3 \mu \mathrm{m}$ in diameter, embedded in a gelatinous matrix (Ford and MacDonald, 1963; Shepard, 1967). Fluorescence microscopy after acridine-orange staining indicates that they are rich in RNA and DNA (Shepard, 1967). Electron microscopy (EM) confirmed these observations and also showed that the cells divide primarily by budding in different directions, producing poly-directional growth ramifications (Shepard, 1969). The outer membrane is trilaminar with a surface layer of hair-like structures (Williams, 1967), and each coccus contains vacuoles and ribosomes, some of which may be arranged in geometrical patterns (Black, Birch-Andersen and Freundt, 1972).

However, some more recent EM studies of thin sections and negatively stained preparations (Black et al., 1972) have described the organisms as short rods and filamentous forms with branches and terminal club-shaped swellings, rather than as spherical bodies embedded in a gelatinous matrix. In an attempt to resolve these differences we have examined the gross morphology and ultrastructure of five laboratory strains isolated from patients with nonspecific urethritis (NSU) (Furness, 1973), by several light- and electron-microscope techniques.

\section{MATERIALS AND METHODS}

The formulae of the T-mycoplasma broth and agar media, T-broth and T-agar (Furness, 1973), and the techniques for preparing stock cultures (Furness, 1969), titrating viable T-mycoplasmas by colony counts (Furness, Pipes and McMurtrey, 1968), and obtaining single-cell suspensions by sonication and filtration through Millipore or Nalgene membranes (Furness, 1969) have all been described before. Deposition of organisms from suspension was always carried out at $14,500 \mathrm{~g}$ for $30 \mathrm{~min}$.

Received 28 Oct. 1974; accepted 22 Nov. 1974.

\footnotetext{
* Present address: Harvard University School of Public Health, Department of Microbiology, 655 Huntington Avenue, Boston, Massachusetts 02115,

Requests for reprints should be sent to G, Furness, Department of Microbiology, New Jersey Medical School, 100 Berger Street, Newark, N.J. 07103, USA.

J. MED. MICROBIOL.-VOL. 8 (1975) 
T-mycoplasmas. The sources of the T-mycoplasmas have already been reported (Furness, 1973); nos. T-960, T-McA, T-27, T-58, and T-Pirillo are laboratory strains, while nos. T-207, T-210, T-213, T-220 and T-221 were recent isolates from patients with non-specific urethritis.

Light Microscopy. The gross morphology of the T-mycoplasmas was observed directly in wet preparations. A drop of T-broth culture or suspension was placed on a microscope slide under a coverslip, the edges of which were sealed with a paraffin-vaseline mixture to prevent evaporation. Specimens were observed at 1000-fold magnification in a Reichert Zetopan microscope by darkfield, phase-contrast or interference (Nomarski) illumination and photographed with a Nikkormat camera.

For photography the specimen was mixed with an equal volume of warm $20 \%(\mathrm{w} / \mathrm{v})$ gelatin Trypticase Soy Broth (BBL). On cooling, the broth formed a clear viscous layer between the coverslip and the slide, eliminating Brownian movement and facilitating continuous observation of individual mycoplasmas in situ.

Electron microscopy. Pellets of T-mycoplasmas were obtained by centrifuging $100-\mathrm{ml}$ amounts of late exponential-phase T-broth cultures containing about $1 \times 10^{6}$ mycoplasmas per $\mathrm{ml}$. Preparations were examined in a Hitachi HU12 electron microscope with an accelerating voltage of $75 \mathrm{KV}$.

Pseudoreplicas. These were made by the method of Furness (1970) and Furness and DeMaggio (1972). Drops of T-mycoplasma suspension or T-broth culture were placed on $1 \%$ Noble agar plates and, after an interval for adsorption, the micro-organisms were fixed by keeping the open plates in air-tight jars above a layer of $40 \%$ formalin for at least 3 days at room temperature $\left(26-27^{\circ} \mathrm{C}\right)$. Blocks containing the drops were cut from the agar and coated with $0.2 \%$ parlodion in ethyl acetate. The pseudoreplicas were stripped off, cut and transferred to grids for EM at $\times 6000$ magnification. These preparations were not shadowed.

Thin sections. Pellets of T-mycoplasmas were suspended in $2 \%$ glutaraldehyde in $0 \cdot 1 \mathrm{M}$ cacodylate buffer and fixed at room remperature for at least $30 \mathrm{~min}$. The fixed cells were centrifuged, and the deposit was washed in Millonig's buffer (Millonig, 1961) and post-fixed for $1 \mathrm{~h}$ in Millonig's buffer containing $1 \%$ osmic acid. The pellet was then dehydrated through alcohols and propylene oxide and finally embedded in epon (Luft, 1961). Thin sections were cut in the grey to silver interference colour range and collected on Formvarcoated 200-mesh copper grids. They were stained for $5 \mathrm{~min}$. with a saturated solution of uranyl acetate in 50\% ethanol (Gibbons and Grimstone, 1960), washed with distilled water, counterstained for 3 min. with lead citrate (Reynolds, 1963), washed again, and then coated lightly with carbon for examination in the EM.

Freeze-fracture and freeze-etched preparations. A pellet of T-mycoplasmas was resuspended in a few $\mathrm{ml}$ of phosphate-buffered saline (PBS) and redeposited in a small centrifuge tube. The PBS was decanted and the pellet treated with $20 \%$ glycerol for $24 \mathrm{~h}$ at $4^{\circ} \mathrm{C}$. A portion of the pellet, about $2 \mathrm{~mm}^{3}$, was placed in a specimen holder and immediately immersed in liquid Freon-12 cooled in liquid nitrogen. When frozen, the specimen holder was inserted into a Hitachi HFZ-1 block precooled in liquid nitrogen and placed in a Hitachi HUS-4GB vacuum evaporator. The specimen was fractured when the vacuum reached $1 \times 10^{-6}$ torr. Etching was performed at $-100^{\circ} \mathrm{C}$ for $10 \mathrm{~min}$., followed by shadowing with platinum, and coating with carbon. The carbon replica was floated off, cleaned with Chlorox, washed in distilled water and placed on a 300-mesh athene grid for examination.

Negatively stained T-mycoplasmas. T-mycoplasmas were resuspended in 1-2 ml of either T-broth or $2 \%$ glutaraldehyde in cacodylate buffer for $30 \mathrm{~min}$. Drops of each suspension were placed on Formvar-coated copper grids, any excess being removed by blotting the edge of the grid. The fixed and unfixed preparations were negatively stained on the grid with a loopful of $1 \%$ molybdic acid adjusted to $p \mathrm{H} 6 \cdot 8-7 \cdot 0$ by ammonium hydroxide.

\section{RESULTS}

\section{The effect of centrifugation on T-mycoplasmas}

Since the T-mycoplasmas were prepared both as pellets and as washed cells, the effect of centrifugal stresses on viability and morphology was examined. 
After ensuring, by light microscopy, that suspensions were single-cell, they were centrifuged over the range of $10,000 \mathrm{~g}$ to $18,000 \mathrm{~g}$ for either $15 \mathrm{~min}$. or $30 \mathrm{~min}$. It was found that the organisms were effectively deposited at $14,500 \mathrm{~g}$ for $30 \mathrm{~min}$., without inactivation; the supernate failed to produce colonies when inoculated on to T-agar, and similar colony counts were obtained from the original single-cell suspension and the deposit after its resuspension by sonic oscillation for 1-2 min. Moreover, light microscopy confirmed that the T-mycoplasmas were not distorted by these manipulations. Therefore, centrifugation at $14,500 \mathrm{~g}$ for $30 \mathrm{~min}$. was used routinely to obtain pellets for electron microscopy and for washing.

\section{Light microscopy}

Darkfield, phase-contrast, and interference microscopy were compared for the useful study of viable T-mycoplasmas in wet preparations of 17-h T-broth cultures. Sterile, uninoculated T-broth medium was also examined by phase-contrast microscopy for the possible presence of T-mycoplasma-like particles. Some batches of T-broth medium did, in fact, contain clumps of spherical particles about the same size as T-mycoplasmas. However, these had no obvious internal structures and they could be disrupted by forces of centrifugation and sonic treatment that failed to affect T-mycoplasmas. Their source was traced to the horse serum, and they were removed by filtration through a Millipore membrane of $0 \cdot 22-\mu \mathrm{m}$ pore diameter. Thereafter, filtered horse serum was used in all experiments involving light microscopy.

\section{Darkfield microscopy}

The flat observation field of darkfield microscopy failed to resolve details of topography, and it was difficult to see the formation of the buds during the early stages of replication of the T-mycoplasmas.

\section{Phase-contrast microscopy}

The cytosol of the T-mycoplasmas was seen by this system. During replication, small dense granules were often observed in the cytoplasm moving between the parent cell and the bud, indicating the absence of an intervening membrane or septum. However, the halo associated with this technique prevented accurate measurement of the size of the micro-organisms.

\section{Interference (Nomarski) microscopy}

Measurement of individual organisms and their buds was readily possible by this technique. The organisms were spherical, with a size range of $0.25-1 \cdot 0 \mu \mathrm{m}$. Their viability was deduced from the presence of budding. All ten strains had the same gross morphology (fig. 1), and there were no obvious morphological differences between unfixed viable micro-organisms and organisms fixed with $2 \%$ glutaraldehyde in $0.1 \mathrm{M}$-cacodylate buffer, except that the latter tended to form aggregates. There was no evidence of a capsule or motility. 
Single cells predominated in exponential phase cultures of strains T-McA, T-Pirillo, T-27, T-58, T-207, T-210, T-220, and T-221, while strains T-213 and $\mathrm{T}-960$ contained mostly aggregates of a few cells.

\section{Pseudoreplication}

Pseudoreplicas of single cells were seen as spherical particles, $0 \cdot 2-1 \cdot 0 \mu \mathrm{m}$ in size, confirming the results obtained by light microscopy. Most cells had a single bud; a few had two (fig. 2).

Colony formation was studied after inoculating drops of single-cell suspension on to T-agar plates. After incubation for 6-7 h and $17 \mathrm{~h}$, plates were randomly selected and the colonies were pseudoreplicated. At 6-7 h, each colony consisted of small clumps of 6-8 cells, some of which were budding. At $17 \mathrm{~h}$, the clumps were larger. The colonies of all 10 strains were similar and the cells were surrounded by a matrix which could be described as capsular material (fig. 3).

\section{Freeze-fracture and freeze-etch}

The examination of viable micro-organisms by freeze-fracture and freezeetch confirmed the results obtained by the other techniques. Additionally, however, fracture and separation of the layers of the trilaminar membrane revealed the granular structure of the inner layers, the granules measuring $7.5-12.5 \mathrm{~nm}$. These account for the rough-textured appearance of the outer surface of the cell (fig. 4). In fig. 5, fracture appears to have exposed the cytoplasm of one organism, although discrete cytoplasmic structures are not discernible. The same figure also demonstrates that some T-mycoplasmas are partially covered with an amorphous material overlying the rough-textured trilaminar membrane.

\section{Thin sections}

In thin sections, too, T-mycoplasmas were observed as spheres $0 \cdot 2-1 \cdot 0 \mu \mathrm{m}$ in size, and the trilaminar membrane, 10-11 nm thick, was covered with short hair- or pilus-like structures, $5-8 \mathrm{~nm}$ long. The internal structure changed as the T-mycoplasmas increased in size. The smallest organisms, $0.2 \mu \mathrm{m}$ in diameter, were densely packed with ribosomes, $12-15 \mathrm{~nm}$ in size, but it was difficult to detect nuclear material. In larger organisms, $0.5 \mu \mathrm{m}$ in diameter, fibrous nuclear strands, 7-9 $\mathrm{nm}$ wide, were distributed throughout the cytoplasm and occasionally small vacuoles were present (fig. 6). The largest organisms were often degenerate or necrotic, with peripherally situated ribosomes and large vacuoles (fig. 7).

The first indication that replication was occurring was the oval appearance of the organism, with slight invagination on each side (fig. 8). Thereafter, the ribsomes concentrated towards each end of the organism and the invagination deepened into the ribosome-free area to form a bud (fig. 9); the division was usually unequal (fig. 10). When the bud was approximately $0.15 \mu \mathrm{m}$ in size, the ribosomes in the parental cell were usually paired and formed polyribosomes consisting of parallel arrangements of three or four chains, each 
MORPHOLOGICAL STUDY OF T-MYCOPLASMAS
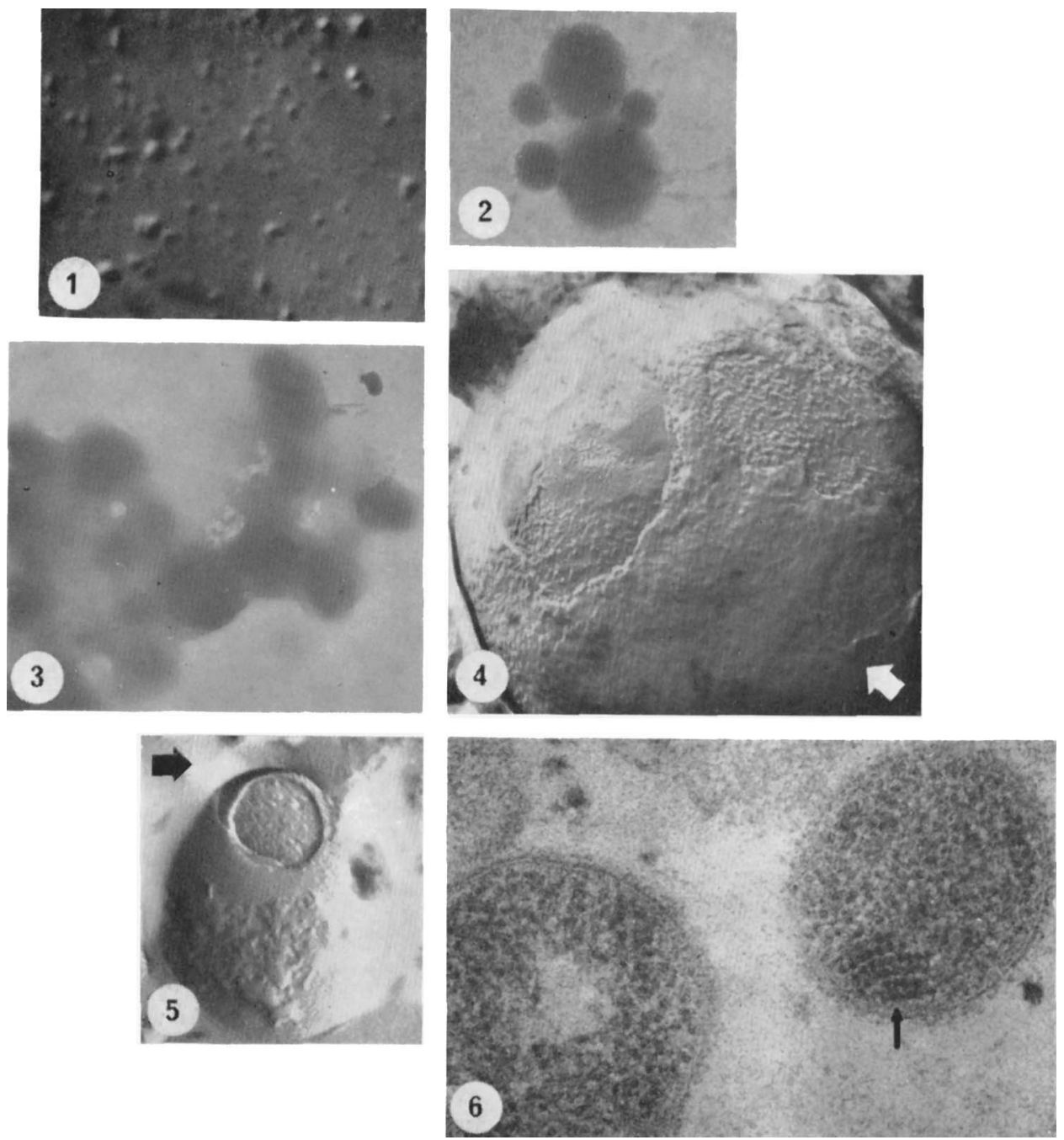

FIG, 1.-Viable T-mycoplasmas, strain T-58, from a 17-h broth culture, showing the essentially single-cell dispersion of the organisms. Interference (Nomarski) microscopy. $\times 3500$.

FIG. 2.-Pseudoreplica of T-mycoplasma, strain T-McA, showing the simultaneous production of two buds by a single parental cell. Electron microscopy (EM). $\times 18,000$

FIG. 3.-Pseudoreplica of a 10-h colony of T-mycoplasma, strain T-960, grown from a single cell. The cells are surrounded by an amorphous matrix. EM. $\times 18,000$.

Fig. 4.-Freeze-etched cell of T-mycoplasma, strain T-58, showing the inner surfaces of the fractured trilaminar membrane. Numerous granular particles are seen that contribute to the rough texture of the outer surface of the cell. The arrow indicates the direction of shadow. EM. $\times 64,000$.

FIG. 5.-Freeze-etched cell of T-mycoplasma, strain T-McA, in which fracture has exposed the cytoplasm. All the surfaces are convex, and an amorphous material partially covers the textured outer membrane. The arrow indicates the direction of shadow. EM. $\times 50,000$.

FIG. 6.-Thin section of T-mycoplasma cells during the exponential phase of broth culture. There are pilus-like projections on the outer surface of the trilaminar membrane, and a vacuole, ribosomes and a geometrically patterned polyribosome (arrowed intracellularly). EM. $\times 80,000$. 

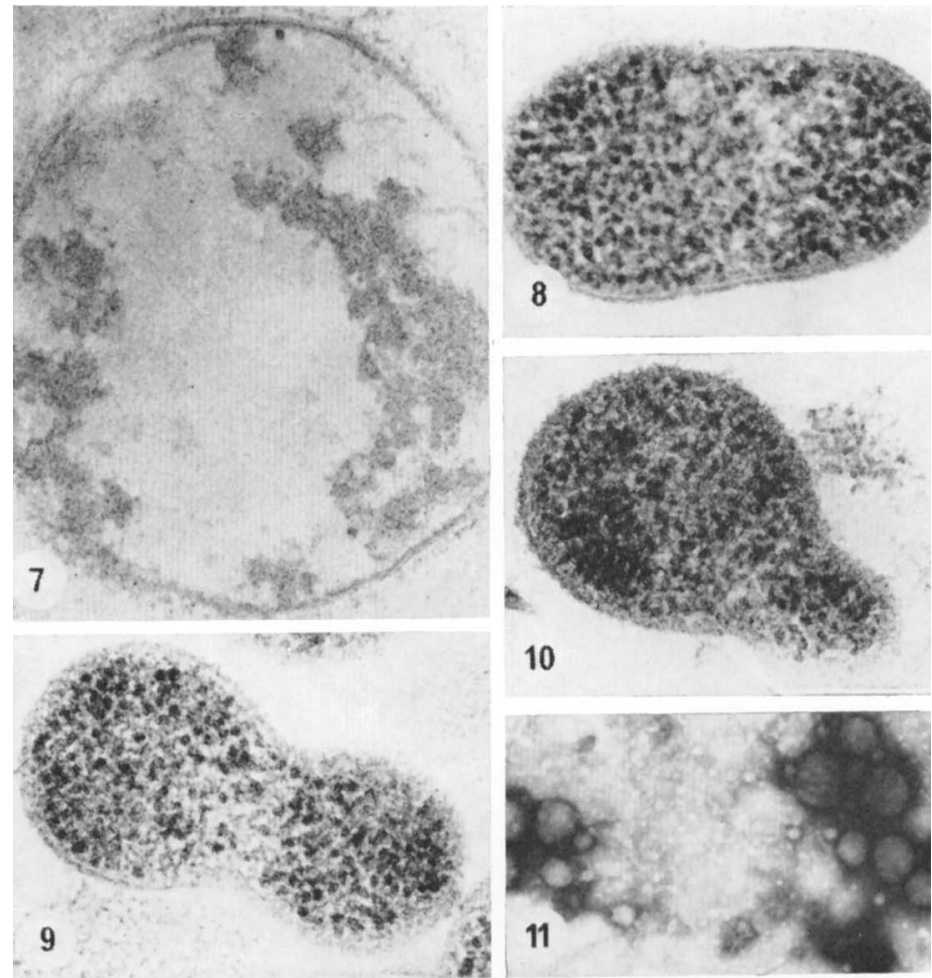

10

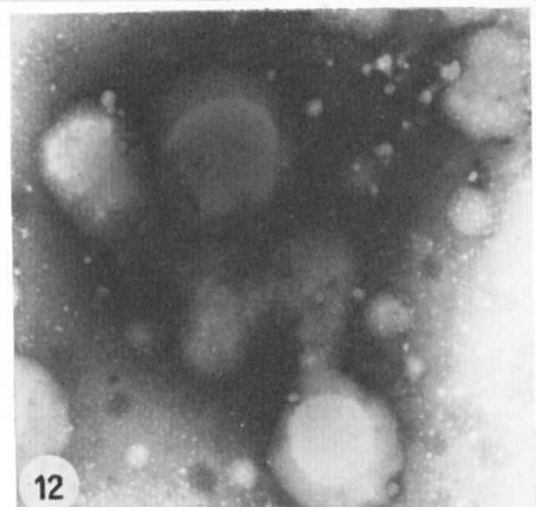

Fig. 7.-Thin section of a large cell of T-mycoplasma, strain T-960, from a broth culture in the decline phase, showing extensive vacuolation and necrosis. EM. $\times 80,000$.

FIG. 8.-Thin section of T-mycoplasma strain, T-Pirillo, illustrating the early stage of budding, with elongation of the cell and slight invagination of the opposing sides. EM. $\times 80,000$.

Frg. 9.-Thin section of a budding cell of T-mycoplasma, strain T-Pirillo, showing more obvious invagination of the outer membrane into the ribosome-free area of cytoplasm. EM. $\times 80,000$.

FIG. 10.-Thin section showing the characteristically unequal division of budding cells, near the end of the replication cycle. The parent cell contains a large polyribosome. EM. $\times 80,000$.

Fig. 11.- Negatively stained and fixed (glutaraldehyde) preparation of T-mycoplasma, strain T-58, during the exponential phase of broth culture. The cells are spherical in shape. EM. $\times 22,500$.

FIG. 12.-Negatively stained preparation of unfixed cells of T-mycoplasma, strain T-58, during the exponential phase of culture. The cells have assumed bizarre shapes and filamentous forms. EM. $\times 30,000$. 
chain being composed of six to eight paired ribosomes (figs. 6 and 10). Progressive invagination of the cell membrane eventually led to separation of the bud. There was no evidence of transverse-septum formation.

\section{Negative staining}

All ten strains of T-mycoplasma, when fixed with glutaraldehyde and negatively stained with molybdic acid, appeared in the EM as typical spheres (fig. 11). On the other hand, viable, unfixed T-mycoplasmas, strains T-58 and T-960, negatively stained, had bizarre and distorted shapes (fig. 12).

\section{Discussion}

We have examined a larger number of human T-mycoplasmas than have others in the past, including four laboratory strains already investigated (Shepard, 1969; Black et al., 1972). Previously, only fixed and stained preparations were studied by light microscopy (Ford and MacDonald, 1963; Shepard, 1967). Viable cells, however, can be examined in wet preparations, by darkfield, phase-contrast and interference microscopy, and both their size and shape were found to be similar to those of fixed cells. On the other hand, we were unable to demonstrate any capsular matrix around viable cells, such as is seen in fixed preparations (Ford and MacDonald, 1963; Shepard, 1967). By darkfield microscopy the cells lacked definition, but both phase-contrast and interference microscopy had individual advantages for studying viable T-mycoplasmas. Phase contrast was ideal for observing the cytosol of the cells during division; dense particles, probably polyribosomes, were seen moving between the parent and daughter cells, indicating the absence of any dividing septum. Only interference microscopy could be used to measure accurately the size of viable cells; this was made technically easier by incorporating gelatin in the suspending fluid to eliminate Brownian movement. Both techniques were useful for confirming the absence of distortion of the Tmycoplasmas during preparative procedures for EM, as well as for detecting mycoplasma contamination in laboratory media or the presence of T-mycoplasma-like artefacts; the latter can be differentiated from mycoplasmas by their lack of internal structure, under phase contrast, and by their ready disintegration by either sonication or centrifugation.

The various EM techniques that we employed confirmed the observations by light microscopy that T-mycoplasmas ranged in size from $0 \cdot 2-1 \mu \mathrm{m}$ and that replication occurred by the production of one or sometimes two buds simultaneously. The absence of artefacts and of changes in gross morphology suggest that the same techniques do not cause significant alterations in the ultrastructure of the organisms. While pseudoreplicas enabled gross morphology to be studied at higher magnification, freeze-fracture and freezeetching revealed that the trilaminar membrane of T-mycoplasmas, like that of other mycoplasmas, contained granular particles, probably of protein (Maniloff and Morowitz, 1972). Thin sections were especially informative 
and confirmed most of the observations of Black et al. (1972), namely that T-mycoplasmas contained DNA strands, ribosomes, polyribosomes and vacuoles, and are bounded by a trilaminar membrane on the outer surface of which are short pilus-like structures. However, whereas Black et al. noted clumping of the ribosomes with the formation of several vacuoles, the ribosomes in our preparations were distributed evenly throughout the cell with only an occasional vacuole. We were able to confirm the striking geometrical patterns of the polyribosomes. Since these were the only intracellular particles large enough to be detected by the diffraction of phase-contrast microscopy, 0.25 $0.3 \mu \mathrm{m}$, it seems reasonable to deduce that they were the small, dense granules observed passing between parent and daughter cells, probably actively engaged in the protein synthesis required for replication of T-mycoplasmas.

In contrast to the thin sections, pseudoreplicas of colonies and small aggregates suggested that $\mathrm{T}$-mycoplasmas produce an amorphous capsular material. Nevertheless, it has not been established that this is a true capsule rather than the outer layer of densely packed pili. If $\mathrm{T}$-mycoplasmas do cause non-specific urethritis (Shepard, 1970) the pili may have pathogenic significance, as in gonococci (Punsalang and Sawyer, 1973).

Unlike Black et al. (1972) we never observed filamentous forms although the same strains were used in both studies. They examined only fixed cells, and the discrepancy can be accounted for by changes in the tonicity of their glutaraldehyde-veronal fixative. Aldehyde fixatives cannot be buffered with veronal acetate because of a Cannizzaro-type reaction; the buffering capacity of the veronal is lost while the aldehyde is reduced to alcohol and salt (Weakley, 1972). It is unlikely, therefore, that the T-mycoplasmas examined by Black et al. were fixed adequately during the lengthy process employed before addition of the osmic-acid fixative. Reuss (1967) has reported that negative staining of unfixed mycoplasmas results in change of morphology. We also observed the formation of filamentous forms of strains T-58 and T-960 when unfixed cells were negatively stained. We conclude, therefore, that the human $\mathrm{T}$ mycoplasmas are normally spherical micro-organisms.

\section{SUMMARY}

The morphology of 10 strains of T-mycoplasma was studied in wet preparations of viable cells by darkfield, phase-contrast and interference microscopy, and in fixed preparations by various techniques of electron microscopy. Mycoplasma-like artefacts in the horse-serum component of the medium were eliminated by filtration. All 10 strains were similar. Individual cells were spherical, $0 \cdot 25-1.0 \mu \mathrm{m}$ in size, with a bounding trilaminar membrane, $10 \mathrm{~nm}$ thick and containing $7 \cdot 5-12 \cdot 5-\mathrm{nm}$ particles, and a layer of pilus-like projections, 5-8 $\mathrm{nm}$ long, on the outer surface. A possible capsular matrix was observed only by the pseudoreplica technique. The cells contained 12-15-nm ribosomes, nuclear fibroids $7 \cdot 5-9 \mathrm{~nm}$ wide, and vacuoles. During replication, the cell elongated slightly and the ribsomes migrated to the ends of the cell leaving a ribosome-free area into which the bounding membrane invaginated to form 
a bud. The bud eventually separated by completion of the process of invagination; a cross-septum did not form. Usually only a single bud developed but sometimes two appeared simultaneously.

The authors wish to thank Miss Marsha Trocola for her very capable technical assistance. This research was supported by NIH Grant No. R01 A108282 from the National Institute of Allergy and Infectious Diseases.

\section{REFERENCES}

Black, F. T., Birch-ANDERSEn, A. AND Freundt, E. A. 1972. Morphology and ultrastructure of human T-mycoplasmas. J. Bact., 111, 254.

Ford, D. K. AND MACDonald, J. 1963. Morphology of human genital "T-strain" pleuropneumonia-like organisms. J. Bact., 85, 649.

FURNESS, G. 1969. Differential responses of single cells and aggregates of mycoplasmas to ultraviolet irradiation. Appl. Microbiol., 18, 360.

FurNess, G. 1970. The growth and morphology of mycoplasmas replicating in synchrony. J. infect. Dis., $122,146$.

FURNESs, G. 1973. T-mycoplasmas: their growth and production of a toxic substance in broth. J. infect. Dis., 127, 9.

FuRNeSs, G. AND DEMAGgIo, M. 1972. Binucleate classical mycoplasmas pathogenic for goats. Infect. Immun., 5, 433.

FurNeSS, G., PIPES, F. J. AND MCMURTREY, M. J. 1968. Susceptibility of human mycoplasmata to ultraviolet and $\mathrm{X}$ irradiations. $J$. infect. Dis., 118, 1 .

GibBons, I. R. AND GRIMSTONE, A. V. 1960. On flagellar structure in certain flagellates. J. biophys. Biochem. Cytol., 7, 697.

LUFT, J. H. 1961. Improvements in epoxy resin embedding methods. J. biophys. biochem. Cytol., 9, 409.

Maniloff, J. AND Morowitz, H. J. 1972. Cell biology of the mycoplasmas. Bact. Rev., 36, 263.

Millonig, G., 1961. Advantages of a phosphate buffer for $\mathrm{OsO}_{4}$ solutions in fixation. $J$. appl. Physics, 32, 1637.

Punsalang, A. P. JR, AND SAwYer, W. D. 1973. Role of pili in the virulence of Neisseria gonorrhoeae. Infect. Immun., 8, 255.

Reuss, K. 1967. Influence of fixation on gross morphology of mycoplasma. J. Bact., 93, 490.

ReYNolds, E. S. 1963. The use of lead citrate at high $p \mathrm{H}$ as an electron-opaque stain in electron microscopy. J. Cell Biol., 17, 208.

SHEPARD, M. C. 1967. Cultivation and properties of T-strains of mycoplasma association with nongonococcal urethritis. Ann. N.Y. Acad. Sci., 143, 505.

ShePARD, M. C. 1969. Fundamental biology of the T-strains. In The Mycoplasmatales and the L-phase of bacteria, edited by L. Hayflick, New York, p. 49.

SHEPARD, M. C. 1970. Nongonococcal urethritis associated with human strains of " $T$ " mycoplasmata. J. Am. med. Ass., 211, 1335.

WEAKLEY, B. S. 1972. A beginner's handbook in biological electron microscopy, Edinburgh, p. 197.

Williams, M. H., 1967. Electron microscopy of T-strains. Ann. N.Y. Acad. Sci., 143, 397. 